\title{
Pulmonary artery trunk enlargement on admission as a predictor of mortality in in-hospital patients with COVID-19
}

\author{
Qing-Qing Zhu ${ }^{1,2} \cdot$ Tao Gong $^{1} \cdot$ Guo-Quan Huang ${ }^{1,3} \cdot$ Zhong-Feng Niu ${ }^{2} \cdot$ Ting Yue $^{2} \cdot$ Fang-Yi Xu $^{2} \cdot$ Chao Chen $^{2}$. \\ Guang-Bin Wang ${ }^{1}$ (D)
}

Received: 19 October 2020 / Accepted: 12 January 2021 / Published online: 22 March 2021

(c) Japan Radiological Society 2021

\begin{abstract}
Purpose To describe the prognostic value of pulmonary artery (PA) trunk enlargement on the admission of in-hospital patients with severe COVID-19 infection by unenhanced CT image.

Materials and methods In-hospital patients confirmed COVID-19 from January 18, 2020, to March 7, 2020, were retrospectively enrolled. PA trunk diameters on admission and death events were collected to calculate the optimum cutoff using a receiver operating characteristic curve. According to the cutoff, the subjects on admission were divided into two groups. Then the in-hospital various parameters were compared between the two groups to assess the predictive value of PA trunk diameter. Results In the 180 enrolled in-hospital patients $(46.99 \pm 14.95$ years; $93(51.7 \%)$ female, 14 patients $(7.8 \%)$ died during their hospitalization. The optimum cutoff PA trunk diameter to predict in-hospital mortality was $>29 \mathrm{~mm}$ with a sensitivity of $92.59 \%$ and a specificity of $91.11 \%$. Kaplan-Meier survival curves for PA trunk diameter on admission showed that a PA trunk diameter $>29 \mathrm{~mm}$ was a significant predictor of subsequent death (log-rank $<0.001$, median survival time of PA $>29 \mathrm{~mm}$ was 28 days).

Conclusion PA trunk enlargement can be a useful predictive factor for distinguishing between mild and severe COVID-19 disease progression.
\end{abstract}

Keywords COVID-19 $\cdot$ Pulmonary artery trunk diameter $\cdot$ Pneumonia $\cdot$ Death events $\cdot$ Prognosis

\section{Introduction}

A novel coronavirus has caused a global outbreak of a respiratory illness termed COVID-19 since December 2019. There were more than 39 million patients with confirmed COVID-19 worldwide by mid-October and more than 1.1 million deaths. One of the key issues has been the very high volume of patients presenting to health centers or

Guang-Bin Wang

wgb7932596@hotmail.com

1 Department of Radiology, Shandong Medical Imaging Research Institute, Cheeloo College of Medicine, Shandong University, No. 324 Jinwu Rd, Jinan 250021, Shandong, China

2 Department of Radiology, Sir Run Run Shaw Hospital, Zhejiang University School of Medicine, No. 3, Qingchun East Rd, Hangzhou 310016, Zhejiang, China

3 Department of Radiology, Wuhu City Second People's Hospital, No. 259 Jiuhua Rd, Wuhu 241000, Anhui, China hospitals during the outbreak. This high demand, especially the need for critical care support, clearly overwhelms the available human and mechanistic capacities. An academician of the American Society for Radiation Oncology called for the immediate establishment of a CT-based diagnostic method for COVID-19 and improvement of the detection rate of SARS-CoV-2 [1]. In such a setting, another solution with sufficient accuracy is needed to guide the quick management of patients who present to the hospital during this pandemic. Thus, early and effective predictors of clinical outcomes are urgently needed for the risk stratification of COVID-19 patients.

The advantages of CT in the diagnosis of COVID-19 are obvious, as this method has great value for early screening, differential diagnosis, and disease severity assessments [2]. While the virus does have a predilection for the lungs, the infection also affects the entire body and seriously affects the cardiovascular system [3]. Pulmonary artery (PA) trunk enlargement is a well-known indicator suggestive of hemodynamic instability, such as acute central pulmonary 
embolism and heart failure [4-6]. The dilation of PA trunk likely reflects cardiac and vascular injury, abnormal blood coagulation in addition to the intrapulmonary inflammation. However, the prognostic value of this parameter and the optimal cutoff of PA trunk diameter on unenhanced CT images to predict mortality have not been well evaluated. The purpose of this study was to describe the prognostic value of pulmonary artery (PA) trunk diameter enlargement on unenhanced CT image in patients with severe COVID-19 infection.

\section{Materials and methods}

\section{Patient cohort}

This was a retrospective study conducted in Jingmen Wuhan Province, China, which was a designated hospital for COVID-19 patients. The inclusion criteria were consecutive adult patients (aged 18 years or older) with laboratory-confirmed COVID-19 who underwent at least one chest CT scan between 18 January 2020 and 7 March 2020 at a single center. The diagnosis of COVID-19 was made according to the World Health Organization interim guidance and confirmed by RNA detection of SARS-CoV-2 in an onsite clinical laboratory. A total of 180 participants were retrospectively screened. The study was approved, and the requirement for informed consent was waived by the ethics commission.

\section{Data collection}

All clinical, CT imaging, laboratory, and outcome data were extracted from electronic medical records using a standardized data collection form.

\section{CT protocol}

All examinations were performed on a GE LightSpeed VCT or on a GE Optima CT660 (General Electric Company, Fairfield, CT, USA). To minimize motion artifacts, patients were instructed on breath-holding; CT images were then acquired during a single breath-hold, axial images were acquired with a $0.625 \mathrm{~mm}$ slice thickness $(120 \mathrm{kVp}, 150 \mathrm{mAs}, 512 \times 512$ matrix) and sagittal and coronal images were reconstructed with a slice thickness of $0.6-0.7 \mathrm{~mm}$.

Image analysis: chest CT patterns of COVID-19 and PA trunk diameter were independently analyzed by three chest radiologists (N.Z.F., C.C. and Y.T. with 15, 10 and 5 years of experience, respectively) on a PACS workstation (Tomtaw International Group, Eword V 5.0.2.43 (×86), China). The readers were blinded to the patients' status as well as the clinical and biological features.
Pulmonary artery trunk diameter was measured of the widest portion of the main pulmonary artery within $3 \mathrm{~cm}$ of the bifurcation (Fig. 1). At some of the time, pulmonary artery bifurcation and the widest portion of the pulmonary trunk do not appear in the same single slice of the CT scan. In this situation, multiplanar reconstruction (MPR) measurement should be used. The measurement must be obtained orthogonal to the long axis of the main pulmonary artery trunk within $3 \mathrm{~cm}$ of the bifurcation in the axial reformatted plane. Discordance was resolved by discussion between the radiologists to reach consensus.

The abnormal radiological findings of $\mathrm{CT}$ reported were performed as in previous studies [7, 8], using terms including ground-glass opacity (GGO), crazy-paving pattern, and consolidation for quantitative evaluation of the progression of COVID-19 pneumonia. The base CT score was assigned according to the extent of GGO involvement in the lobes (with a maximum score of 5 possible for each of the five lobes), which scores defined as follows: 0 , no involvement; $1,<5 \%$ involvement; $2,5-25 \%$ involvement; $3,26-49 \%$ involvement; 4, 50-75\% involvement; and 5, > 75\% involvement. Weight assignments were different for the three categories of CT findings (i.e., GGO, crazy-paving pattern, and consolidation). If the crazy-paving pattern appeared in one lobe, the base CT score was increased by 1 , and if consolidation appeared (either with or without the crazypaving pattern), the base CT score was increased by 2 . Therefore, a maximum CT score of 7 was possible for each lobe. The total CT score was the sum of the individual lobar scores and ranged from 0 (no involvement) to 35 (maximum involvement), with the highest possible CT score indicating

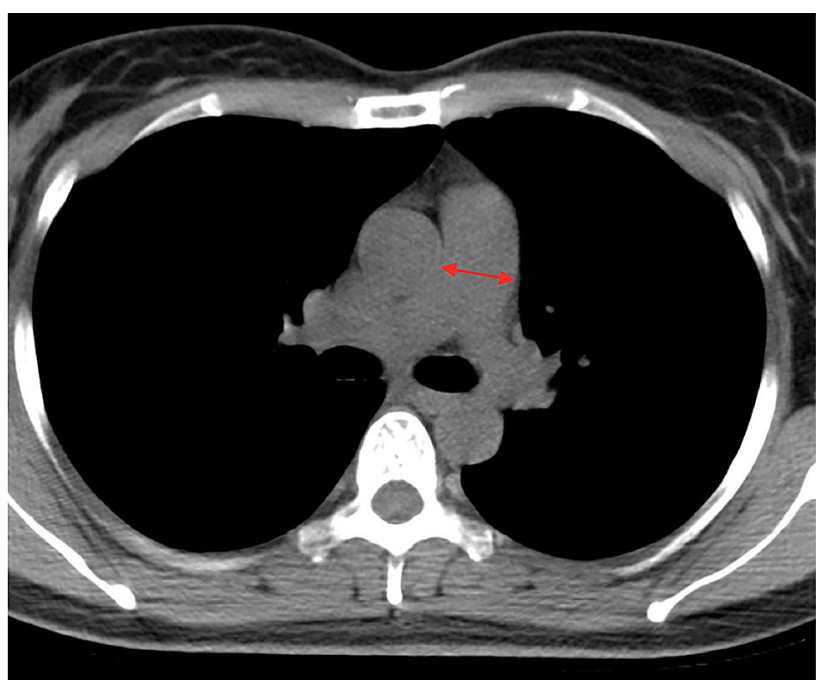

Fig. 1 Pulmonary artery trunk measurement on axial Chest CT Imaging. The measurement (red line) must be obtained in the axial plane, orthogonal to the long axis of the main pulmonary artery within $3 \mathrm{~cm}$ of the bifurcation 
consolidation in all five lobes (Figs. 2, 3). Details regarding the evaluation of lobe involvement may be found in previous study by Ooi et al. [9].

Unenhanced MDCT was performed on every in-hospital patient on admission. Blood samples were also collected after admission for routine laboratory tests, such as C-reaction protein, procalcitonin and coagulation profile, and serum biochemical tests (including renal and liver function) performed in an onsite laboratory.

\section{Statistical analysis}

Continuous and categorical variables are presented as the median \pm standard deviation or median (interquartile range, IQR), as appropriate. Categorical variables are presented as n (\%). Event frequencies were compared with the chisquare test. Other comparisons between two groups were made with unpaired Student $t$ test or the Mann-Whitney $U$ test. Then we used both univariate analysis and multivariate regression analysis to test for this association after proportional hazard assumption using Schoenfeld's method. For multivariate analysis, factors achieving $P<0.10$ in univariate analysis were used a forward stepwise procedure for estimating hazard ratios (HRs) and 95\% CIs. Multivariate
Cox regression analysis proved that PA trunk diameter is an independent prognostic factor for adverse outcomes. The PA trunk diameter cutoff point was evaluated by receiver operator characteristic (ROC) curve. The outcomes were compared by Kaplan-Meier survival analysis. The hazard ratio (HR) and 95\% confidential interval (95\% CI) were calculated by log-rank tests. A $P<0.05$ was accepted as statistically significant. All statistical analyses were performed using IBM SPSS version 23.0 (IBM SPSS Statistics, IBM Corporation, Armonk, NY) and MedCalc (MedCalc 15.2.2 version, MedCalc Inc., Mariakerke, Belgium).

\section{Results}

\section{Baseline characteristics and establishing an optimum cutoff value for PA trunk diameter on admission}

The 180 eligible patients had a median age of 47 years (IQR 35-57 years), ranging from 18 to 90 years. In total, $41 / 180$ patients were older than 57 years. 93 patients were female. Comparison of basic clinical characteristics of the in-hospital patients on admission (the first time in hospital),

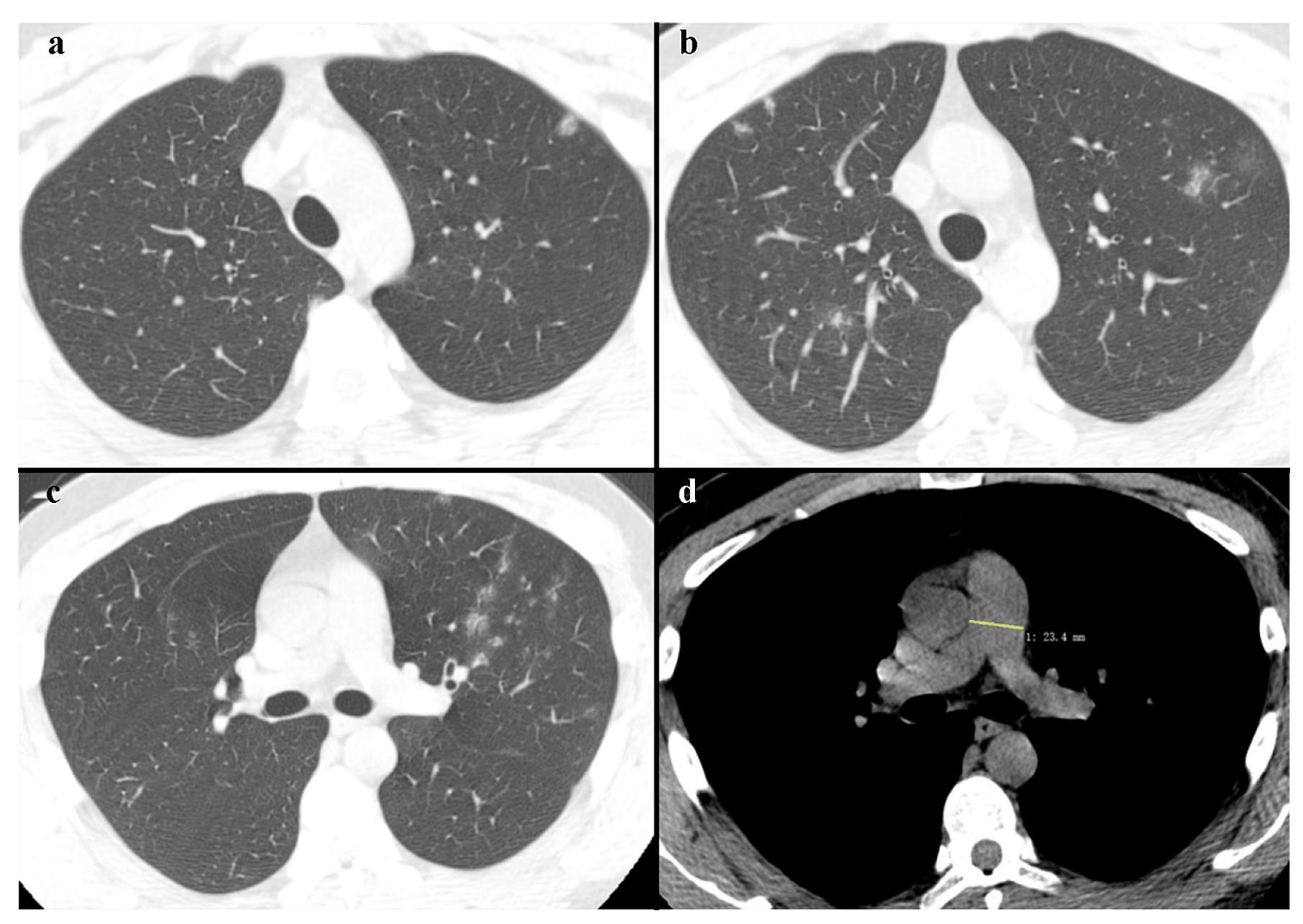

Fig. 2 27-Year-old man with coronavirus disease pneumonia in the mild condition on admission. a-c Ground-glass opacity (GGO) was observed in two upper lobes and right middle lobe on admission, for total CT score of 7. d The diameter of the pulmonary trunk was $\leq 29 \mathrm{~mm}$ 


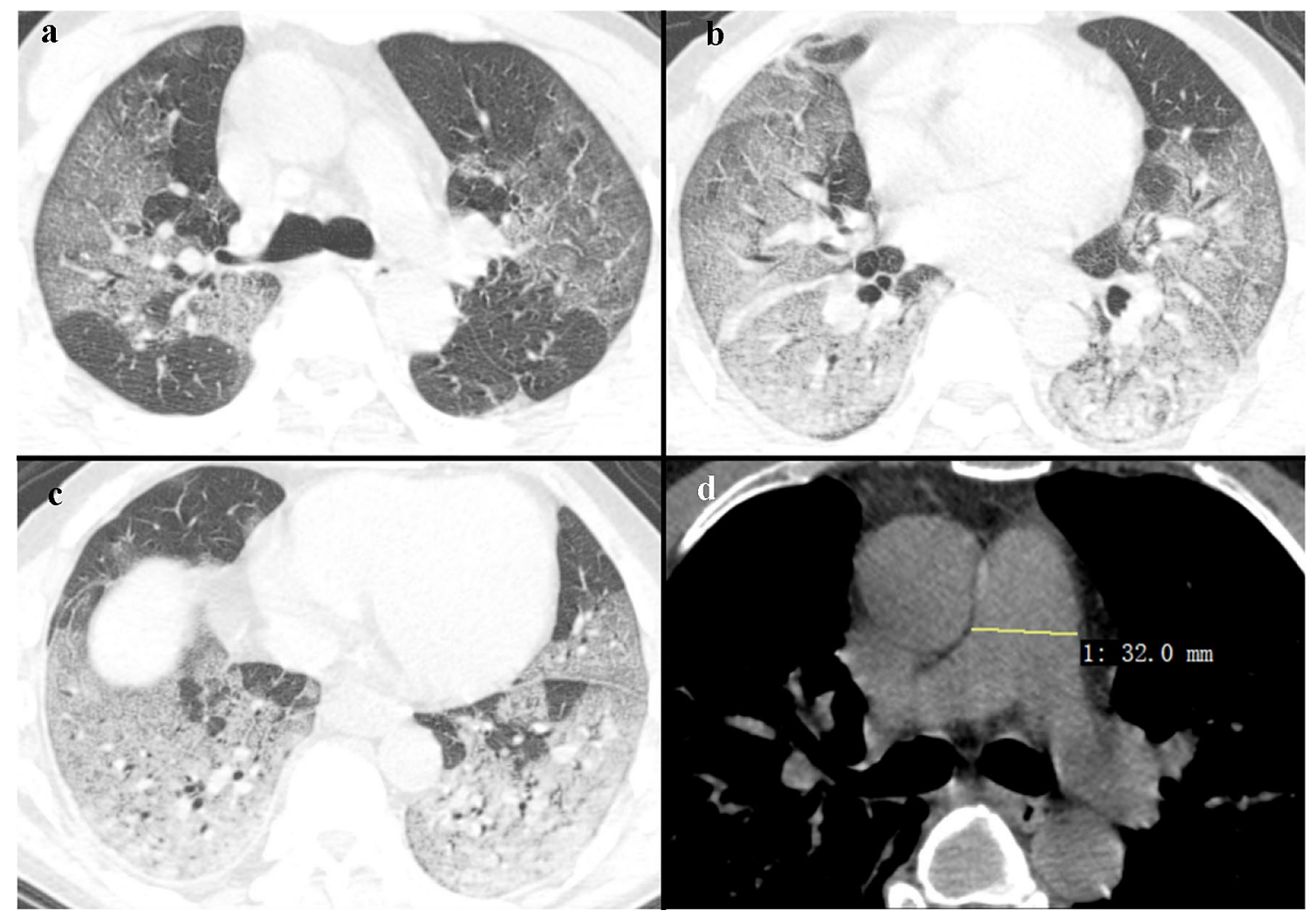

Fig. 3 50-Year-old man with coronavirus disease pneumonia in the severity condition on admission. a-c Severe pulmonary parenchyma consolidation with partial crazy-paving pattern was observed on

including age, sex, previous history, between survior and non-survior is shown in Table 1 . A total of 14 all-cause deaths occurred during hospitalization, and there were 166 survivors; The first time CT score and PA trunk diameters were significantly different between the survior and non-survior. To identify predictors for death, we performed a univariate Cox regression analysis followed by a multivariate Cox regression analysis in Table 2. CRP and PA trunk diameter on admission were independent risk factors. The optimum cutoff value (0.965) for PA trunk diameter was obtained by ROC curve (Fig. 4), with a sensitivity of $92.59 \%$ and a specificity of $91.11 \%$. Patients in this study were divided according to PA trunk diameter (PA $>29 \mathrm{~mm}$ and PA $\leq 29 \mathrm{~mm}$ ). There were significant differences between groups for most variables (Table 3 ). Compared to patients with PA trunk diameters $\leq 29 \mathrm{~mm}$, patients with PA trunk diameters $>29 \mathrm{~mm}$ had a higher incidence of underlying diseases, such as diabetes, cardio-cerebrovascular diseases, and chronic kidney disease $(P<0.001)$. There were differences between the two groups in the incidence of underlying diseases, such as diabetes $(P<0.001)$, coronary heart disease $(P<0.001)$ and chronic kidney disease $(P<0.001)$. Additionally, higher levels of LDH $(P<0.001)$, CRP $(P<0.001)$, D-dimer $(P<0.001)$, PCT $(P<0.001)$ and lower levels of admission, for total CT score 30 . d The diameter of the pulmonary trunk was $>29 \mathrm{~mm}$ used MPR measurement

lymphocyte $(P<0.001)$ were observed in patients with PA trunk diameters $>29 \mathrm{~mm}$.

\section{Dilated PA trunk as a predictor of mortality}

Kaplan-Meier survival curves (Fig. 5) for PA diameter on admission showed that a PA trunk diameter $>29 \mathrm{~mm}$ was a significant predictor of subsequent death (logrank $P<0.001$, median survival time of PA $>29 \mathrm{~mm}$ was 28 days).

\section{Discussion}

In this retrospective study, a PA trunk diameter greater than $29 \mathrm{~mm}$ on admission was identified as an independent predictor of severe disease. This study presented a well-established cutoff value to predict adverse outcomes in patients with COVID-19. Current guidelines [10] recommend performing unenhanced chest CT to assess for COVID-19 CT patterns. However, CT should not be performed only as a screening test in patients with or without symptoms. CT may also be helpful in patients with moderate and severe symptoms. Prior reports [11] have suggested that myocarditis and 
Table 1 Baseline characteristics of 180 in-hospital patients with COVID-19 on admission between survivor and non-survivor

\begin{tabular}{|c|c|c|c|c|}
\hline Variable & Total $(n=180)$ & Survivor $(n=166)$ & Non-survivor $(n=14)$ & $P$ value \\
\hline Age (years) & $46.99 \pm 14.95$ & $45.87 \pm 14.43$ & $60.36 \pm 15.05$ & $<0.001$ \\
\hline Female, $n(\%)$ & $93(51.7 \%)$ & $86(51.8 \%)$ & $7(50.0 \%)$ & 0.558 \\
\hline CT score & $14.12 \pm 8.79$ & $26.29 \pm 7.46$ & $13.10 \pm 8.11$ & $<0.001$ \\
\hline PA trunk diameter & $25.80 \pm 3.81$ & $33.2 \pm 3.76$ & $25.2 \pm 3.10$ & $<0.001$ \\
\hline \multicolumn{5}{|l|}{ Underlying condition } \\
\hline Diabetes (type 2), $n(\%)$ & $14(7.8 \%)$ & $9(5.4 \%)$ & $5(35.7 \%)$ & 0.002 \\
\hline Cardio-cerebrovascular disease, $n(\%)$ & $36(20 \%)$ & $25(15.1 \%)$ & $11(78.6 \%)$ & $<0.001$ \\
\hline Chronic kidney disease, $n(\%)$ & $10(5.6 \%)$ & $2(1.2 \%)$ & $8(57.1 \%)$ & $<0.001$ \\
\hline COPD & $2(1.1 \%)$ & $2(1.2 \%)$ & $0(0.0 \%)$ & 0.850 \\
\hline \multicolumn{5}{|l|}{ Routine text on admission } \\
\hline LDH (U/L) (IQR) & $186.5(160,235.5)$ & $182.50(157.75,226.00)$ & $307.00(277.00,490.25)$ & $<0.001$ \\
\hline CK-MB (U/L) & $10.15 \pm 5.90$ & $9.96 \pm 5.75$ & $12.43 \pm 7.35$ & 0.133 \\
\hline CRP (mg/L) (IQR) & $8.85(3.00,18.10)$ & $7.65(2.60,14.85)$ & $86.65(45.68,95.62)$ & $<0.001$ \\
\hline PCT (ng/L) (IQR) & $0.05(0.03,0.08)$ & $0.04(0.03,0.07)$ & $1.05(0.13,5.74)$ & $<0.001$ \\
\hline D-dimer (mg/L) (IQR) & $0.355(0.210,0.692)$ & $0.300(0.210,0.617)$ & $1.170(0.700,4.575)$ & $<0.001$ \\
\hline Lymphocyte $\left(10^{9} / \mathrm{L}\right)(\mathrm{IQR})$ & $1.205(0.853,1.530)$ & $1.25(0.928,1.583)$ & $0.505(0.340,0.685)$ & $<0.001$ \\
\hline Neutrophil $\left(10^{9} / \mathrm{L}\right)(\mathrm{IQR})$ & $2.86(1.953,4.060)$ & $2.67(1.935,3.963)$ & $4.16(2.588,7.703)$ & 0.033 \\
\hline
\end{tabular}

Data are the mean $\pm \mathrm{SD}$, median (IQR), or $n(\%) . P$ values were calculated with the $t$ test, Mann-Whitney $U$ test, or $\chi^{2}$ test, as appropriate $I Q R$ interquartile range, $P A$ pulmonary artery, $C O P D$ chronic obstructive pulmonary disease, $C R P$ including 4 missing survivor data), $P C T$ including 12 missing survivor data, $D$-dimer including 2 missing non-survivor and 20 survivor data, $L D H$ lactate dehydrogenase, $C K$ - $M B$ creatine kinase isoenzymes, $C R P$ C-reaction protein, $P C T$ procalcitonin

Table 2 Univariate and stepwise multivariate Cox hazard analysis for future adverse events

\begin{tabular}{|c|c|c|c|c|c|c|c|c|}
\hline \multirow[t]{2}{*}{ Variable } & \multicolumn{4}{|c|}{ Univariate analysis } & \multicolumn{4}{|c|}{ Multivariate analysis } \\
\hline & $\beta$ coefficient & SE & $P$ & HR & $\beta$ coefficient & SE & $P$ & HR $(95 \% \mathrm{CI})$ \\
\hline Lymphocyte (109/L) & -4.805 & 1.106 & $<0.001$ & 0.008 & & & & \\
\hline $\mathrm{LDH}(\mathrm{U} / \mathrm{L})$ & 0.006 & 0.001 & $<0.001$ & 1.006 & & & & \\
\hline CK-MB & 0.037 & 0.038 & 0.332 & 1.037 & & & & \\
\hline CRP & 0.057 & 0.009 & $<0.001$ & 1.058 & 0.038 & 0.10 & $<0.001$ & $1.039(1.018-1.061)$ \\
\hline CT score & 0.171 & 0.041 & $<0.001$ & 1.187 & & & & \\
\hline PA diameter & 0.501 & 0.08 & $<0.001$ & 1.650 & 0.380 & 0.094 & $<0.001$ & $1.462(1.216-1.757)$ \\
\hline Age (years) & 0.055 & 0.017 & 0.002 & 1.057 & & & & \\
\hline Cardio-cerebrovascular disease & 2.740 & 0.659 & $<0.001$ & 15.481 & & & & \\
\hline Chronic kidney disease & 3.914 & 0.585 & $<0.001$ & 50.113 & & & & \\
\hline Diabetes (type 2) & 1.825 & 0.601 & 0.002 & 6.205 & & & & \\
\hline
\end{tabular}

Variables (on admission) with $P<0.1$ in univariate analysis were applied to a multivariate analysis

$S E$ standard error, $H R$ hazard ratio

coagulopathy are associated with COVID-19 infection and ultimately predict a more unfavorable prognosis. The above laboratory data indicate that the group with PA trunk diameters $>29 \mathrm{~mm}$ on admission has statistically more serious inflammation and heart injuries, consistent with literature reports of patients with severe disease. The study by Grillet et al. [12] points to a high prevalence of acute pulmonary embolism in patients with severe COVID-19. Additional contrast-enhanced $\mathrm{CT}$ and echocardiography may be required in patients with pulmonary embolism and myocarditis. Creel-Bulos et al. [13] analyzed five SARS-CoV-2-positive ICU patients with acute right ventricular dilatation and impaired systolic function, showing that additional contrastenhanced CT is indicated for patients with suspected complications. However, the use of contrast is contraindicated in certain patients, such as those with renal insufficiency, and excessive use of multidetector contrast-enhanced test (such as CT pulmonary angiography (CTPA)) may result in 
Fig. 4 Receiver operator characteristic curve of pulmonary trunk measurements for predicting deaths. The optimum cutoff point, identified as the point closest to the upper left corner, was $29 \mathrm{~mm}$, with a $92.59 \%$ sensitivity and $91.11 \%$ specificity. The area under the receiver operator characteristic curve was 0.96

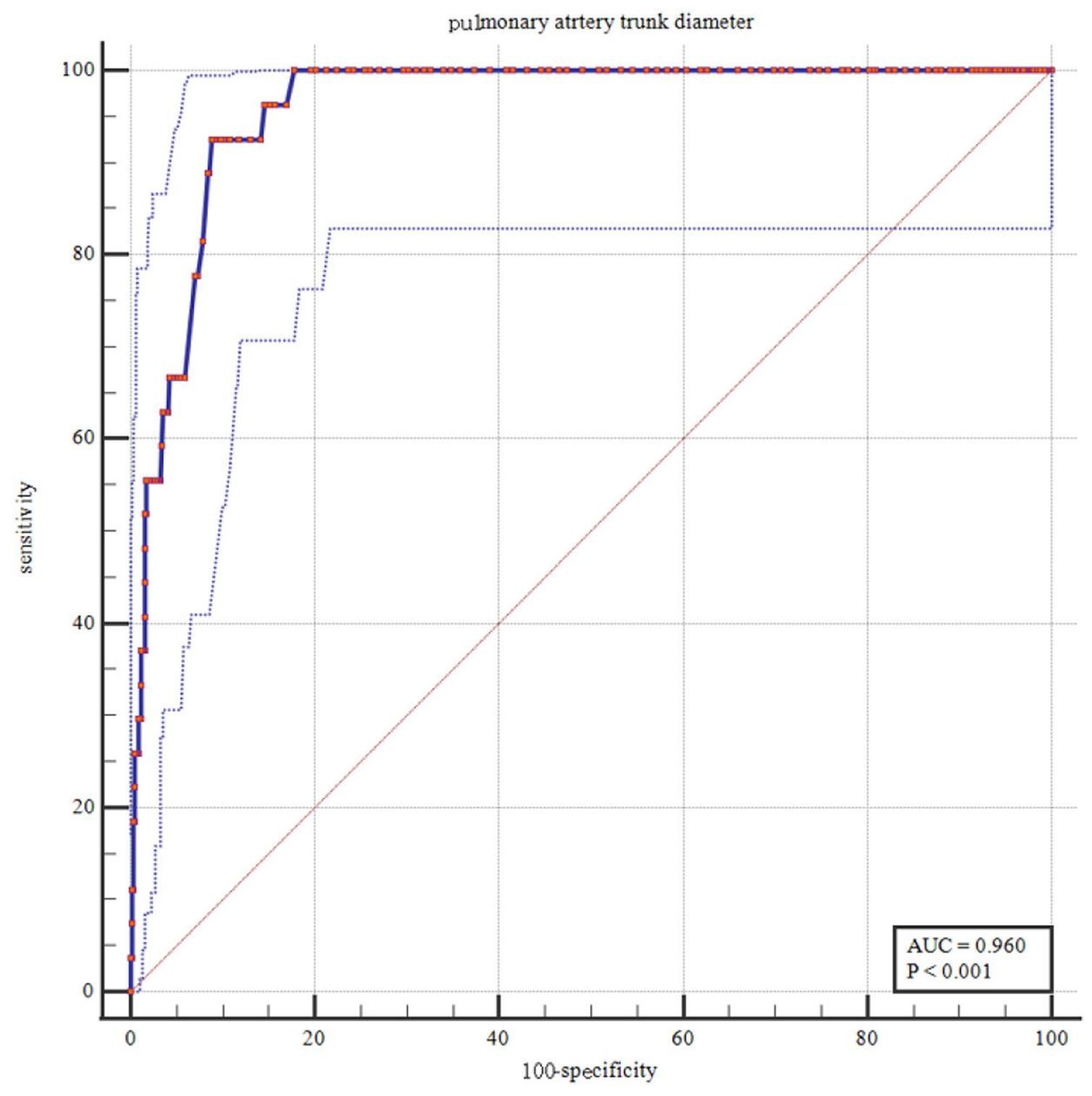

excessive radiation exposure. Unenhanced MDCT may offer a rapid indication of the degree of severity. Rapid diagnosis of the degree of severity has been shown to reduce the mortality rate. Therefore, we focused on other structures on unenhanced CT, such as the mediastinum, vasculature, heart and pleura, that might be used as alternative signs to predict the outcome. It is important that radiologists establish an accurate diagnosis of severity based on unenhanced MDCT data as soon as possible. In the current study, a clear cutoff value (PA trunk diameter $>29 \mathrm{~mm}$ ) for PA trunk diameter was well established by ROC curve analysis. Notably, of the 14 non-survivors with PA trunk diameters $>29 \mathrm{~mm}, 4$ had no severe symptoms on admission. Thus, for patients who have a large PA trunk diameter, admission to the hospital and close monitoring should be considered even in the absence of other severe symptoms.

A dilated PA trunk indicated an urgent need for care in patients with COVID-19, which might be attributed to several reasons, as follows. First, pro-inflammatory cytokines mediate an inflammatory response and cause lung parenchyma abnormalities, and edema formation disturbs alveolar gas exchange and causes hypoxemia. In parallel, pulmonary vascular resistance increases in response to pro-inflammatory mediators, resulting in increased right ventricular afterload and pulmonary trunk dilation [3]. Second, hypoxemia can stimulate thrombosis in patients with severe disease. Pulmonary embolus was the direct cause of right ventricular failure and pulmonary trunk dilation [11]. The direct sign of pulmonary embolism is pulmonary artery distension. Hospitalized patients, especially patients with severe COVID-19, were more likely to have underlying conditions, long-term bed rest and invasive treatment, which are all risk factors for hypercoagulation or thrombosis. Third, viral infection may be the most common cause of myocarditis, which shows a rapid onset and dramatically accelerated progression towards severe heart failure. The virus infects host cells through angiotensin-converting enzyme 2 (ACE2) receptors, which can lead to acute myocardial injury. Some patients with severe disease may rapidly develop impaired heart pump function and have symptoms of passive pulmonary congestion and even acute respiratory distress syndrome (ARDS). Fourth, laboratory data provide an essential contribution to clinical decision making in this and many other infectious diseases. It was well confirmed in our data that in the 
Table 3 Baseline characteristics of 180 in-hospital patients with COVID-19 on admission between PA groups

\begin{tabular}{|c|c|c|c|c|}
\hline Variable & Total $(n=180)$ & PA diameter $\leq 29(n=151)$ & PA diameter $>29(n=29)$ & $P$ value \\
\hline Age, years (IQR) & $47(35.0,56.8)$ & $45(34.0,56.0)$ & $56(43.5,71.5)$ & 0.022 \\
\hline Female, $n(\%)$ & $93(51.7)$ & $75(49.7)$ & $18(62.1)$ & 0.221 \\
\hline CT score-first time & $14.12 \pm 8.79$ & $13,16 \pm 8.03$ & $19.14 \pm 10.80$ & 0.006 \\
\hline Highest CT score & $14.86 \pm 8.85$ & $13.74 \pm 8.00$ & $20.69 \pm 10.75$ & 0.002 \\
\hline Unilateral or bilateral pleural effusion, $n(\%)$ & $14(7.8)$ & $4(2.6)$ & $10(34.5)$ & $<0.001$ \\
\hline Pericardial effusion, $n(\%)$ & $11(6.1)$ & $6(4.0)$ & $5(17.2)$ & 0.006 \\
\hline Mediastinal lymphadenopathy, $n(\%)$ & $12(6.7)$ & $5(3.3)$ & $7(24.1)$ & $<0.001$ \\
\hline \multicolumn{5}{|l|}{ Underlying condition } \\
\hline Diabetes (type 2), $n(\%)$ & $12(6.7)$ & $6(4.0)$ & $6(20.7)$ & 0.001 \\
\hline Cardio-cerebrovascular disease, $n(\%)$ & $35(19.8)$ & $23(15.2)$ & $12(41.4)$ & 0.001 \\
\hline Chronic kidney disease, $n(\%)$ & $10(5.6)$ & $2(1.3)$ & $8(27.5)$ & 0.001 \\
\hline COPD & 2 & 1 & 1 & - \\
\hline \multicolumn{5}{|l|}{ Routine test } \\
\hline LDH (U/L) (IQR) & $246.9(146.00,219.00)$ & $168(144,207.5)$ & $224(166,328)$ & $<0.001$ \\
\hline CK-MB (U/L) & $10.15 \pm 5.90$ & $9.99 \pm 5.93$ & $11 \pm 5.8$ & 0.399 \\
\hline CRP (mg/L) (IQR) & $12.08(1.50,10.05)$ & $3.3(1.50,8.40)$ & $10.4(2.75,55.45)$ & $<0.001$ \\
\hline PCT (ng/L) (IQR) & $0.04(0.03,0.07)$ & $0.04(0.03,0.06)$ & $0.095(0.475,2.50)$ & $<0.001$ \\
\hline D-dimer (mg/L) (IQR) & $0.46(0.24,0.96)$ & $0.405(0.23,0.775)$ & $1(0.65,6.79)$ & $<0.001$ \\
\hline Lymphocyte $\left(10^{9} / \mathrm{L}\right)(\mathrm{IQR})$ & $1.27 \pm 0.57$ & $1.32 \pm 0.547$ & $0.99 \pm 0.59$ & 0.003 \\
\hline Neutrophil $\left(10^{9} / \mathrm{L}\right)$ (IQR) & $3.46 \pm 2.432$ & $3.39 \pm 2.28$ & $3.79 \pm 3.12$ & 0.418 \\
\hline
\end{tabular}

Data are the mean \pm SD, median (IQR), or $n(\%)$. $P$ values were calculated with the $t$ test, Mann-Whitney $U$ test, or $\chi^{2}$ test, as appropriate

$I Q R$ interquartile range, $L D H$ lactate dehydrogenase, $C K-M B$ creatine kinase isoenzymes, $C R P$ C-reaction protein, $P C T$ procalcitonin, $C R P$ including 4 missing data in PA $\leq 29 \mathrm{~mm}$ group, $P C T$ including 11 missing data in PA $\leq 29 \mathrm{~mm}$ group and 1 missing data in PA $>29 \mathrm{~mm}$ group, $D$-dimer including 18 missing data in PA $\leq 29 \mathrm{~mm}$ group and 4 missing data in PA $>29 \mathrm{~mm}$ group

Fig. 5 Kaplan-Meier survival curves for PA trunk diameter on admission showed that a PA trunk diameter $>29 \mathrm{~mm}$ was a significant predictor of subsequent death (log-rank $P<0.001$, median survival time of $\mathrm{PA}>29 \mathrm{~mm}$ was 28 days)

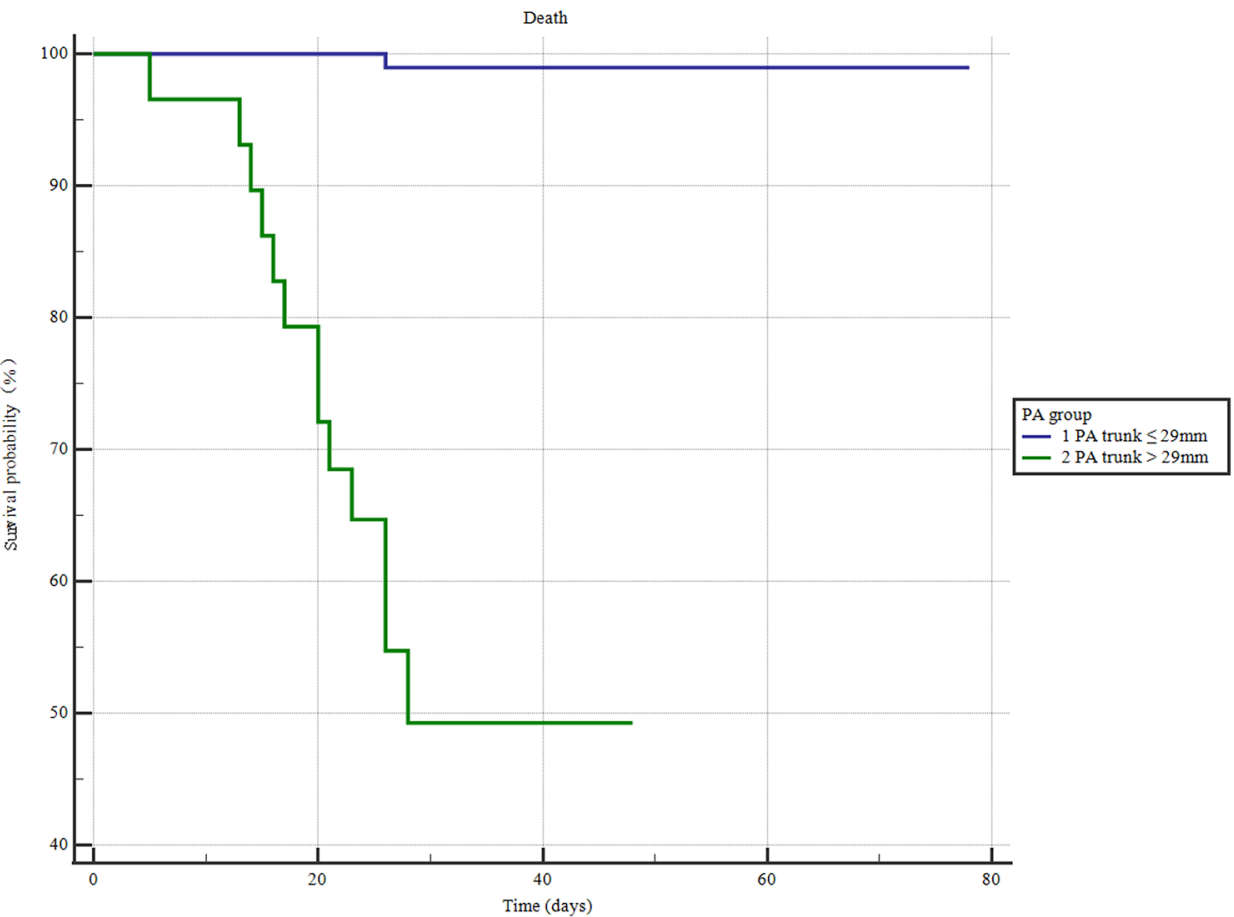


group with enlarged PA trunks, CRP, PCT, and LDH were frequently elevated. In particular, serial PCT is a potential biomarker of bacterial superinfection (either pulmonary or systemic), but these values do not substantially change in patients with viral infections. PCT was found to be associated with a nearly fivefold increased risk for severe disease [14]. In the group with PA trunk enlargement, the substantial increase in PCT would reflect myocardial infection and/ or bacterial coinfection. There were obvious differences between the two groups in the incidence of underlying disease. Elderly patients with comorbidities are at a higher risk for COVID-19, especially those with coronary heart disease, hypertension, chronic kidney disease or diabetes. Pericardial effusion and pleural effusion were uncommon imaging findings in these patients, but patients with severe disease and a PA trunk diameter $>29 \mathrm{~mm}$ showed higher incidences of these conditions than ordinary patients, which is consistent with the literature reports [15].

This study has several limitations. First, even though our study had sufficient power to detect significant differences in mortality between groups, it might have selection bias because this was a single-center, retrospective study. Second, due to differences in access to medical resources, the duration from illness onset to admission for the included patients might not be representative, which might influence the PA trunk measurements. Third, a multiple-parameter prediction model including PA measurements and other variables might provide better predictive ability for COVID-19 patients. Fourth, this study does not describe the progression of PA trunk changes as the patient progresses to severe disease. Dynamic measurement of the PA trunk will reveal more information. The purpose of this study was to explore markers of severe disease in COVID-19 patients. Accordingly, the results from this study demonstrate that a PA trunk $>29 \mathrm{~mm}$ is a predictor for in-hospital death.

In conclusion, a PA trunk diameter $>29$ mm could effectively predict in-hospital mortality in patients with COVID19 , indicating that PA trunk diameter could be an early and helpful marker to improve the management of COVID-19 patients.

Author contributions QZ and GW had full access to all the data in the study and take responsibility for the integrity of the data and the accuracy of the data analysis. Conceptualization: QZ, TG, GH, and GW; methodology: Q, TG, GH, and GW; formal analysis and investigation: QZ, ZN, CC, and TY; writing-original draft preparation: QZ, TG, and GH; writing - review and editing: QZ, GH, and FX; funding acquisition: FX and CC; resources: FX, CC, and TY; supervision: GW, $\mathrm{QZ}, \mathrm{GH}$, and TG.

Funding This research was supported by Zhejiang University special scientific research fund for COVID-19 prevention and control under Grant no. 2020XGZX051.

\section{Compliance with ethical standards}

Conflict of interest The authors declare that they have no conflict of interest.

Ethics approval This article does not contain any studies with human participants or animals performed by any of the authors.

Human rights All procedures performed in studies involving human participants were in accordance with the ethical standards of the institutional and/or national research committee and with the 1964 Declaration of Helsinki and its later amendments or comparable ethical standards.

Informed consent All patients gave their written informed consent for the use of anonymous personal data extracted from their medical records for research purposes.

\section{References}

1. Garg M, Prabhakar N, Gulati A, Agarwal R, Dhooria S. Spectrum of imaging findings in pulmonary infections. Part 1: bacterial and viral. Pol J Radiol. 2019;84:e205-13.

2. Dai WC, Zhang HW, Yu J, et al. CT imaging and differential diagnosis of COVID-19. Can Assoc Radiol J. 2020;71:195-200.

3. Brack MC, Lienau J, Kuebler WM, Witzenrath M. Cardiovascular sequelae of pneumonia. Curr Opin Pulm Med. 2019;25(3):257-62.

4. Chien $\mathrm{CH}$, Shih FC, Chen CY, Chen $\mathrm{CH}$, Mak CW. Unenhanced multidetector computed tomography findings in acute central pulmonary embolism. BMC Med Imaging. 2019. https://doi. org/10.1186/s12880-019-0364-y.

5. Aluja Jaramillo F, Gutierrez FR, Díaz Telli FG, Yevenes Aravena S, Javidan-Nejad C, Bhalla S. Approach to pulmonary hypertension: from CT to clinical diagnosis. Radiographics. 2018;38:357-73.

6. Tan RT, Kuzo R, Goodman LR, Siegel R, Haasler GR, Presberg $\mathrm{KW}$. Utility of CT scan evaluation for predicting pulmonary hypertension in patients with parenchymal lung disease. Chest. 1998;113(5):1250.

7. Bernheim A, Mei X, Huang M, et al. Chest CT findings in coronavirus disease-19 (COVID-19): relationship to duration of infection. Radiology. 2020;295(3):200463.

8. Wu J, Wu X, Zeng W, et al. Chest CT findings in patients with coronavirus disease 2019 and its relationship with clinical features. Invest Radiol. 2020;55(5):257-61.

9. Ooi GC, Khong PL, Muller NL, et al. Severe acute respiratory syndrome: temporal lung changes at thin-section CT in 30 patients. Radiology. 2004;230(3):836-44.

10. Revel MP, Parkar AP, Prosch H, et al. COVID-19 patients and the radiology department-advice from the European Society of Radiology (ESR) and the European Society of Thoracic Imaging (ESTI). Eur Radiol. 2020;30:4903-9.

11. Aghagoli G, Marin BG, Soliman LB, Sellke FW. Cardiac involvement in COVID patients: risk factors, predictors, and complications: a review. J Cardiac Surg. 2020. https://doi.org/10.1111/ jocs. 14538 .

12. Grillet F, Behr J, Calame P, Delabrousse E. Acute pulmonary embolism associated with COVID-19 pneumonia detected by pulmonary CT angiography. Radiology. 2020;296(3):201544.

13. Creel-Bulos C, Hockstein M, Amin N, Melhem S, Sharifpour M. Acute cor pulmonale in critically Ill patients with Covid-19. New Engl J Med. 2020;382(21):e70. 
14. Lippi G, Pleban M. Procalcitonin in patients with severe coronavirus disease 2019 (COVID-19): a meta-analysis. Clin Chim Acta. 2020;505:190-1.

15. Morrill HJ, Pogue JM, Kaye KS, Laplante KL. Treatment options for carbapenem-resistant enterobacteriaceae infections. Open Forum Infect Dis. 2015;2(2):v50.
Publisher's Note Springer Nature remains neutral with regard to jurisdictional claims in published maps and institutional affiliations. 